Check for updates

Cite this: RSC Adv., 2019, 9, 26327

\title{
Flow modification enhancing the growth rate in top seeded solution growth of $\mathrm{SiC}$ crystals $\uparrow$
}

\begin{abstract}
Minh-Tan Ha, (D) ab Yeong-Jae Yu, ${ }^{a}$ Yun-Ji Shin, ${ }^{a}$ Si-Young Bae, ${ }^{a}$ Myung-Hyun Lee, ${ }^{a}$ Cheol-Jin Kim ${ }^{\mathrm{b}}$ and Seong-Min Jeong (D) *a

In this study, multiphysics simulations were carried out to understand the convection mechanisms of the top seeded solution growth (TSSG) of SiC. Experimental melting tests and crystal growth were conducted to verify the simulation results in the growing temperatures between 1700 and $1900{ }^{\circ} \mathrm{C}$ with rf induction heating furnace. From the solidified melt of $\mathrm{Si}-\mathrm{Cr}$ solution after the melting test, the melt flow in the simulation was successfully verified. In the given experimental conditions, the electromagnetic convection was found to govern the global fluid flow, while other mechanisms including the Marangoni convection, the buoyancy convection and the centrifugal forced convection influence the fluid flow near the crystal. Based on an understanding of the fluid flow obtained with the simulations, a structural flow modifier (FM) was applied to enhance the growth rate of the SiC crystal. The growth rates of $\mathrm{SiC}$ with/without FM were successfully estimated from simulations showing good agreements with the experimental values. After the experimental crystal growth using FM, a remarkable enhancement in the growth rate was found in an FM configuration, which suggests a way to improve the growth rate by the TSSG method based on the efficient use of the dissolved $C$ in the melt.
\end{abstract}

Received 30th June 2019

Accepted 15th August 2019

DOI: $10.1039 / c 9 r a 04930 d$

rsc.li/rsc-advances a reasonable growth rate such that TSSG is now assumed as the next-generation fabrication method of SiC crystals.

Because SiC forms incongruent melts of Si with dissolved C, the composition ratio of $\mathrm{Si}$ and $\mathrm{C}$ is inevitably not uniform in the melt of the TSSG method. ${ }^{9}$ When SiC is formed at the crystal, the same amount of $\mathrm{Si}$ and $\mathrm{C}$ is consumed from the melt. Because $\mathrm{C}$ has low solubility in Si liquid, however, $\mathrm{C}$ can deplete easily near the crystal without a continuous $\mathrm{C}$ feed by fluid flow. Hence, the crystal growth of SiC is affected significantly by mass transport in the TSSG method. The mass transport in the melt is inevitably affected by the operating conditions, such as the hot-zone design, operating temperature, and melt composition.

To stabilize the process condition, the mass transport of the melt in the TSSG method should be optimized, as emphasized in previous studies. ${ }^{\mathbf{1 0 - 1 3}}$ Concerning the mass transport of the melt in the TSSG method, Yamamoto et al. reported that both electromagnetic and Marangoni convections are dominant, ${ }^{\mathbf{1 0 , 1 1}}$ while Mercier et al. suggested that buoyancy convection is dominant. $^{12}$ The difference in the dominant factor reported by Yamamoto et al. and Mercier et al. might be due to the differences in experimental setups, including the reactor designs and operating conditions. The difference in the governing convections reported in the literature ${ }^{\mathbf{1 0 - 1 2}}$ suggests that the experimental setup of TSSG should be fully understood for optimizing the crystal growth of SiC.

Because the mass transport of the melt is also influenced by the structural modification of the melt reservoir, efforts to
† Electronic supplementary information (ESI) available: FE-SEM image and EDS element mappings of a cross-sectioned solidified melt of $90 \% \mathrm{Si}-10 \% \mathrm{Cr}$. See

DOI: $10.1039 /$ c9ra04930d
${ }^{a}$ Energy and Environmental Division, Korea Institute of Ceramic Engineering and Technology, Jinju-si 52851, Korea.E-mail: smjeong@kicet.re.kr

${ }^{b}$ Department of Ceramic Engineering, Gyeongsang National University, Jinju-si 52828, Korea 
optimize the mass transport by changing the crucible shape have increased. Mercier et al. suggested a podium crucible design to reduce the destabilizing effect of electromagnetic and Marangoni convections. ${ }^{13}$ Kusunoki et al. adopted an immersion guide attached to a crucible for a homogeneous melt flow near the seed and discovered an improvement in growth rate. ${ }^{\mathbf{1 4}}$ However, the mass transport of the melt in TSSG must be fully understood for the given experimental setup before modifying the crucible shape.

In this study, we aim to obtain an efficient flow modification to enhance the growth rate of SiC by structural flow modification. Therefore, a comprehensive analysis of the mass transport of the melt based on the TSSG method was first conducted using multiphysics simulations. Next, based on further understanding the mass transport of the melt, a structural modification adopting a "flow modifier (FM)" was suggested to enhance the growth rate of SiC. Finally, the effect of the FM on the growth rate was analyzed by comparing the simulation and experimental results.

\section{Experimental and modeling}

The SiC seed crystal was placed by contacting the Si melt with dissolved $\mathrm{C}$ in the induction heating furnace (Takeuchi Electric Co., Japan), as illustrated in Fig. 1a. The operating frequency of the induction-heating furnace was $8.5 \mathrm{kHz}$. High purity (11Nine) Si chunk (OCI, Korea) was loaded into a graphite crucible of inner diameter $50 \mathrm{~mm}$. The amount of $\mathrm{Si}$ was weighed carefully to obtain a Si melt height of $17 \mathrm{~mm}$. The inner crucible was placed inside an outer crucible, where both crucibles were fabricated using graphite (M-501, Morgan, Korea). The crucibles were placed on a crucible shaft that could change the vertical position. A seed crystal was attached to a seed shaft. The graphite parts were enclosed in a thermal insulator (M-501, Morgan, Korea) and isolated in a stainless chamber that was filled by an inert gas such as Ar or He. The temperatures on the melt surface and beneath the outer crucible were measured using pyrometers (IR-CA, Chino, Japan). The melt was heated to a target temperature measured at the melt surface. At the target temperature, the seed crystal touched the melt surface and subsequently rotated at a rate of $30 \mathrm{rpm}$. After growing for $1 \mathrm{~h}$, the seed shaft was lifted to separate from the melt surface. To characterize the grown crystals, the residual Si on the as-grown crystal was removed chemically with a mixed solution of nitric acid and hydrofluoric acid. After the chemical cleaning, the grown crystals were evaluated using optical microscopy (ME600L, Nikon, Japan), field emission scanning electron microscopy (JSM-7610F, Jeol, Japan), energy dispersive spectroscopy (X-Max 50, Oxford Instruments, UK), and microRaman spectroscopy (Alpha300, WITec, Germany). The thickness of the grown layer was measured by optical microscopy after the cross-sectional cutting of the grown crystals following all other characterizations.

Fig. 1b shows the meshed TSSG model used in this study. Meshing is essential in finite element analysis, and an extremely fine mesh is required to solve fluid dynamics because the mesh size affects the calculation accuracy directly. In the

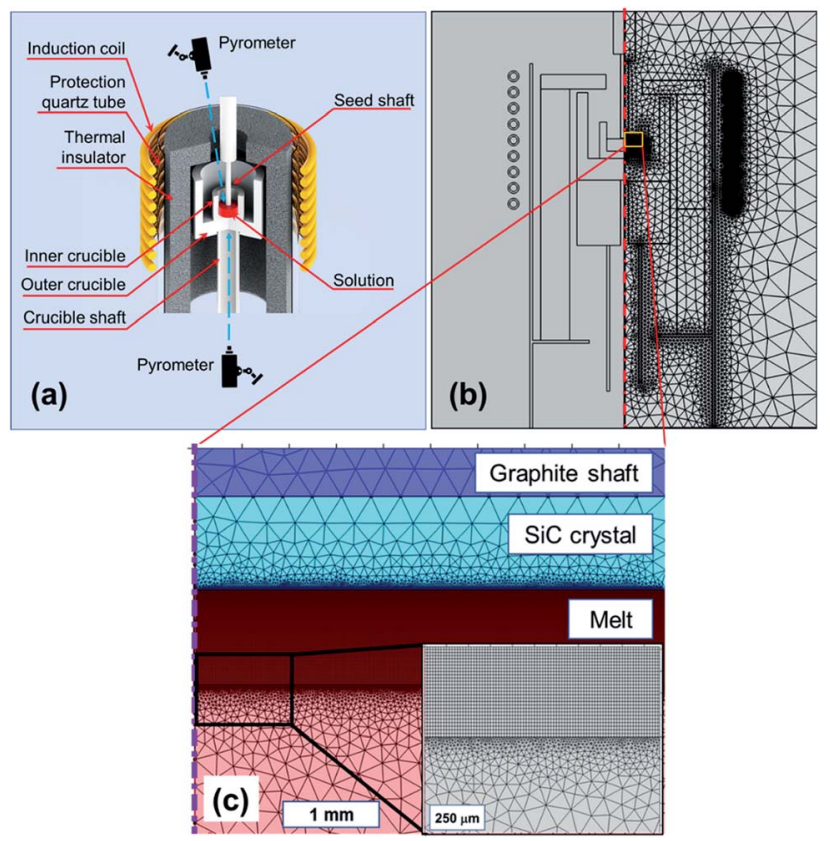

Fig. 1 (a) Schematics of a TSSG grower, (b) global meshed model for TSSG grower, and (c) detailed mesh model in the melt and near crystal/ melt interface (inset).

global domains, the maximum size in the hot-zone was $0.5 \mathrm{~mm}$. To improve the accuracy of the fluid calculation, a $1 \mathrm{~mm}$ layer beneath the seed-solution interface was meshed by rectangular elements of element size of $12.5-500 \mu \mathrm{m}$, as shown in Fig. 1c.

The variables hereinafter are listed in Table 1 . In the induction heating, an induced eddy current is generated according to Lenz's law; subsequently, parts are heated by that current owing to Joule's effect. The electromagnetic field is defined by Maxwell's equation,

$$
\begin{gathered}
\nabla \times H=J \\
B=\nabla \times A \\
E=-j \omega A \\
J=\sigma E+j \omega D .
\end{gathered}
$$

The heat transfer via thermal conduction is calculated as follows:

$$
\rho C_{\mathrm{p}} \cdot \nabla T=\nabla \cdot(k \nabla T)+Q
$$

Thermal radiation is a major mechanism of heat transfer, especially at high temperatures, which is described by the following equations:

$$
\begin{gathered}
G=G_{\mathrm{m}}(J)+F_{\mathrm{amb}} \sigma T_{\mathrm{amb}}^{4} \\
\left(1-\varepsilon_{\mathrm{s}}\right) G=J-\varepsilon \sigma T^{4} \\
\varepsilon_{\mathrm{s}}\left(G-\sigma T^{4}\right)=-n \cdot(-k \nabla T) .
\end{gathered}
$$


Table 1 Nomenclature

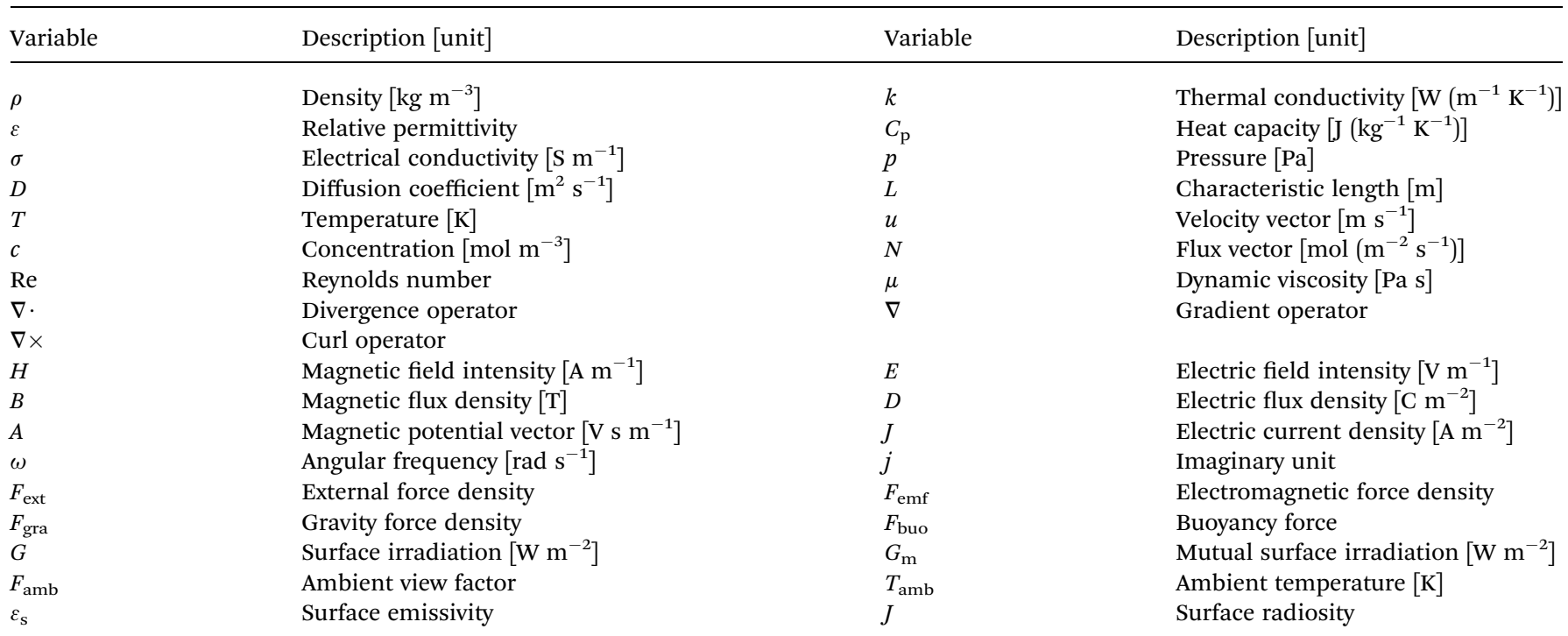

Table 2 Material properties used in the simulation

\begin{tabular}{|c|c|c|c|c|c|c|}
\hline \multicolumn{2}{|l|}{ Properties } & \multirow{2}{*}{$\begin{array}{l}\mathrm{Ar} \\
520.33\end{array}$} & \multirow{2}{*}{$\begin{array}{l}\text { Thermal insulator } \\
200\end{array}$} & \multirow{2}{*}{$\begin{array}{l}\text { Graphite } \\
710\end{array}$} & \multirow{2}{*}{$\frac{\mathrm{SiC}}{1400}$} & \multirow{2}{*}{$\frac{\mathrm{Si}(\text { melt) }}{908.7}$} \\
\hline Heat capacity, $C_{\mathrm{p}}$ & {$\left[\mathrm{J}\left(\mathrm{kg}^{-1} \mathrm{~K}^{-1}\right)\right]$} & & & & & \\
\hline Density, $\rho$ & {$\left[\mathrm{kg} \mathrm{m}^{-3}\right]$} & $522.21 / T$ & 120 & 1950 & 3160 & $3120.45-0.35 T$ \\
\hline Relative permeability, $\mu_{\mathrm{r}}$ & & 1 & 1 & 1 & 1 & 1 \\
\hline Electrical conductivity, $\sigma$ & {$\left[\mathrm{S} \mathrm{m}^{-1}\right]$} & 1 & 430 & 75400 & 225 & $1.2 \times 10^{6}$ \\
\hline Thermal conductivity, $k$ & {$\left[\mathrm{~W}\left(\mathrm{~m}^{-1} \mathrm{~K}^{-1}\right)\right]$} & 0.07 & 0.336 & 150 & 61 & 63 \\
\hline Temperature derivative of the surface & {$\left[\mathrm{N} \mathrm{m}^{-1} \mathrm{~K}^{-1}\right]$} & - & - & - & - & $0.25 \times 10^{-3}$ \\
\hline
\end{tabular}

In this study, the Si melt with dissolved $\mathrm{C}$ was assumed as an incompressible newtonian fluid with laminar flow, following a previous study. ${ }^{13}$ From the literature, ${ }^{10,13}$ we considered four primary convection mechanisms in the model: Buoyancy convection, Marangoni convection, electromagnetic convection, and centrifugal forced convection, by rotating the seed shaft.

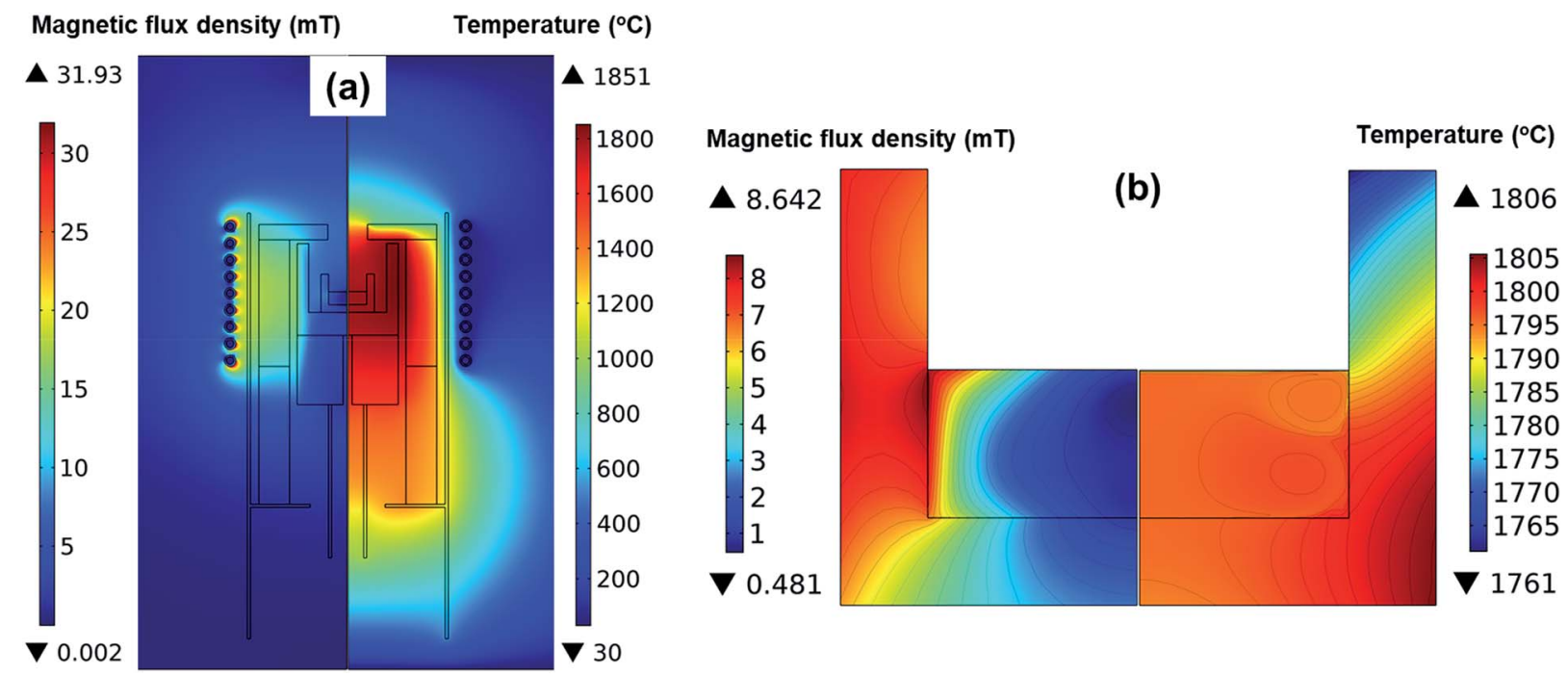

Fig. 2 Magnetic flux density and temperature distribution (a) in the entire reactor, and (b) detailed in the crucible and melt. 
Table 3 Calculated and measured temperatures at the melt free surface and bottom of the outer crucible at three ranges of temperature

\begin{tabular}{|c|c|c|c|c|c|c|}
\hline \multicolumn{2}{|c|}{ (1) Melt surface temperature $\left({ }^{\circ} \mathrm{C}\right)$} & \multicolumn{2}{|c|}{$\begin{array}{l}\text { (2) Crucible bottom } \\
\text { temperature }\left({ }^{\circ} \mathrm{C}\right)\end{array}$} & \multicolumn{2}{|c|}{$\begin{array}{l}\text { Temperature difference } \\
(1)-(2)\left({ }^{\circ} \mathrm{C}\right) \\
\end{array}$} & \multirow[b]{2}{*}{ Error } \\
\hline Experimental & Simulation & Experimental & Simulation & Experimental & Simulation & \\
\hline 1695 & 1694.8 & 1674 & 1674.1 & 21 & 20.7 & $1.43 \%$ \\
\hline 1776 & 1776.4 & 1754 & 1754.2 & 22 & 22.2 & $1.00 \%$ \\
\hline 1889 & 1887.2 & 1865 & 1863.4 & 24 & 23.6 & $1.67 \%$ \\
\hline
\end{tabular}

Therefore, the governing equation of the fluid dynamics is expressed by the following Navier-Stokes equation:

$$
\rho\left(\frac{\partial u}{\partial t}+u \cdot \nabla u\right)=\nabla[-p+\tau]+F_{\text {ext }},
$$

where $\tau=\mu\left(\nabla u+(\nabla u)^{T}\right)$ is the viscous stress, and the external volume force $F_{\text {ext }}=F_{\text {gra }}+F_{\text {emf }}$ is the sum of external forces acting on the melt. The buoyancy convection is a result of nonuniform gravity force density $F_{\text {gra }}=\rho g$ in the melt, owing to the difference in melt density $\rho$ in the non-isothermal melt. The electromagnetic convection is a result of Lorentz force applied to the melt, $F_{\text {emf }}=J \times B$.

The Marangoni effect that is caused by surface tension depends on the tangential temperature gradient $\nabla_{\tau} T$ at the free surface of the melt. ${ }^{11,13}$ Therefore, the viscous force at the free surface is described by the boundary condition $-p+\tau=\gamma \nabla_{\tau} T$, where $\gamma$ is the temperature derivative of the surface tension.
Because of the seed rotation, the boundary condition of the melt velocity at the melt/crystal interface is $u=v_{\mathrm{s}} \varphi$, where $v_{\mathrm{s}}$ is the magnitude of the velocity and $\varphi$ is a vector indicating the out-ofplane direction of flow. The slip condition was applied on the melt surface: $n \cdot u=0$ and $\tau-(\tau \cdot n) n=0$, where $n$ is a vector normal to the boundary. No slip condition was applied to the solution at the solution/crucible interface: $u=0$.

The mass transport of $\mathrm{C}$ in Si melt is driven by diffusion and convection, as described by $\nabla \cdot(-D \nabla c)+u \cdot \nabla c=0$, and the flux of C is defined by $N=-D \nabla c+u \cdot \nabla c$. The solubility of C in Si $C_{0}$ is derived from the temperature-dependent $\mathrm{C}$ fraction in molten Si $x_{\mathrm{C}}$ :

$$
C_{0}=\frac{\rho_{\mathrm{Si}}}{M_{\mathrm{Si}}} \frac{x_{\mathrm{C}}}{1-x_{\mathrm{C}}},
$$

where $x_{\mathrm{C}}=\exp (6.249-24460 / T)$ is obtained from the literature. $^{15,16}$ The equilibrium condition is supposed to be at the

(a)

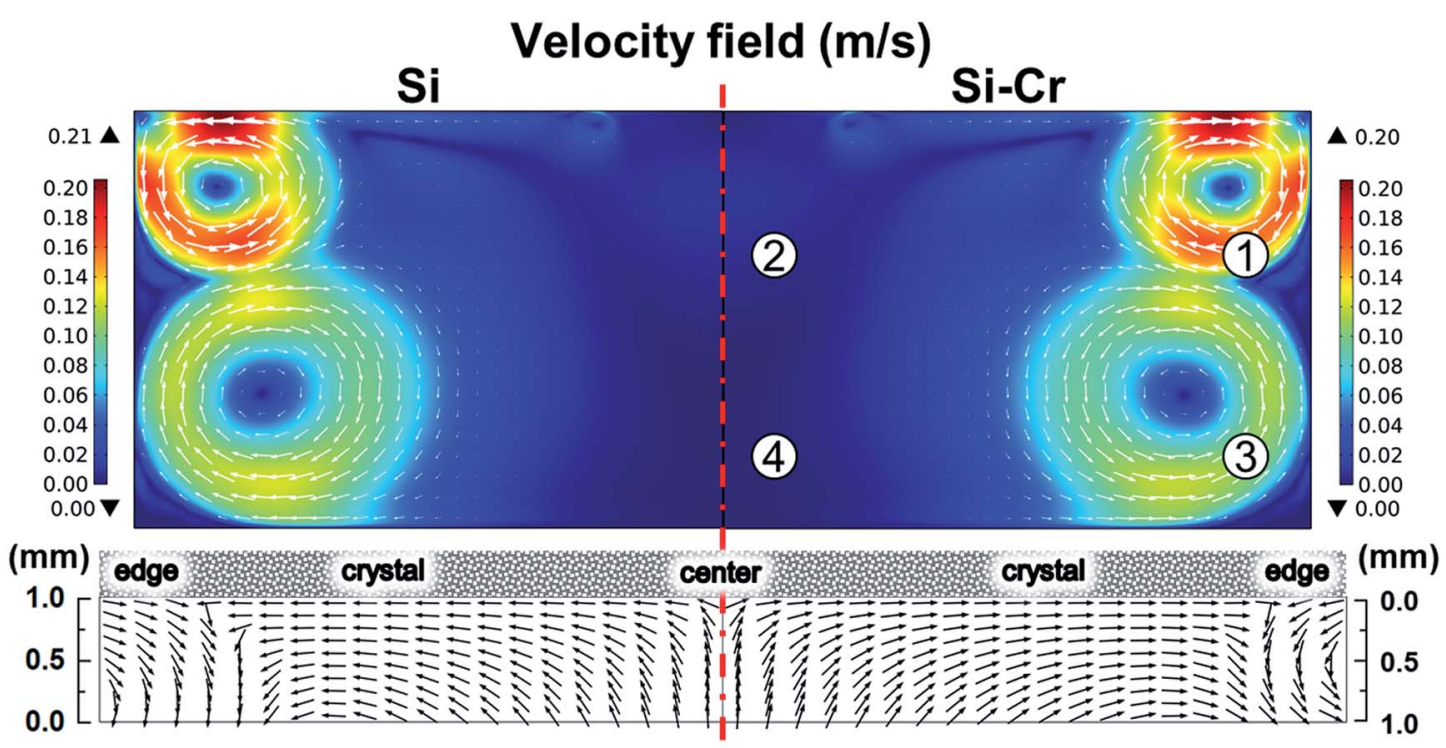

(c)
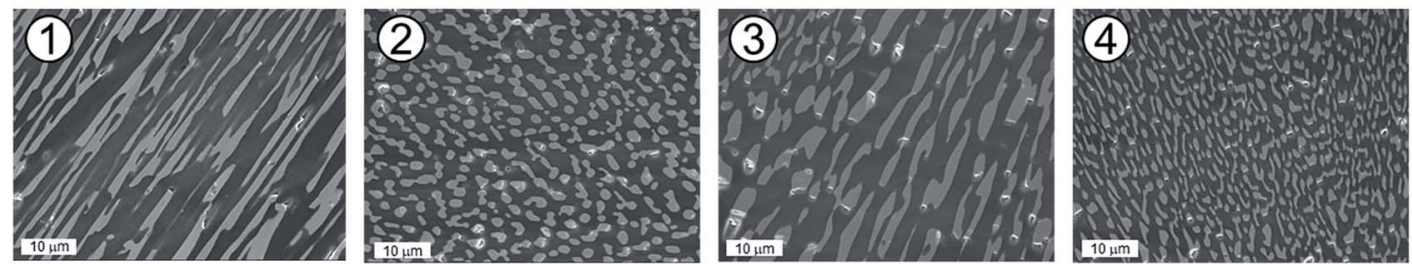

Fig. 3 (a) Simulated velocity field in pure Si melt and Si-Cr melt, (b) simulated fluid flow near the melt/crystal interface, and (c) cross-sectional FE-SEM images for the solidified melt at indicated points. 
interaction interfaces of melt/graphite and melt/crystal; therefore, a boundary condition $C=C_{0}$ was applied on the interfaces of melt/crucible and melt/crystal. A no-flux constraint was applied on the melt surface. The material properties used in the simulation are listed in Table 2 .

\section{Understanding of mass transport of TSSG}

Fig. 2 shows the simulation results of the magnetic flux and the temperature in the reactor when the surface temperature of the melt was $1800{ }^{\circ} \mathrm{C}$. According to Fig. 2a, the highest temperature was $1870{ }^{\circ} \mathrm{C}$ at the wall of the outer crucible. As shown in Fig. 2b, the magnetic field was stronger as it approached the coils, while the magnetic field around the inner crucible was weak. Hence, we assumed that the inductive current was strong in the outer crucible and that the inductive heat generated in the outer crucible was the primary heat source in the reactor. The inner crucible was assumed to be heated by heat transfer primarily from the outer crucible. This could explain the higher temperature near the melt/crucible compared to that at the center of the melt.

To verify the simulation model, a melting test with pure $\mathrm{Si}$ was performed seed crystal at $\sim 1700{ }^{\circ} \mathrm{C}, \sim 1800{ }^{\circ} \mathrm{C}$, and $\sim 1900{ }^{\circ} \mathrm{C}$ with the temperature at the melt surface. Table 3 shows the measured temperatures at the melt surface and bottom of the crucible. The errors between the simulation and experiments were estimated from the temperature difference between the two measured temperature points. The temperature differences are in the range of $21-24{ }^{\circ} \mathrm{C}$, and the errors are approximately $1 \%$, indicating a good agreement between simulation and experiment. (a)

$\Delta 0.025$
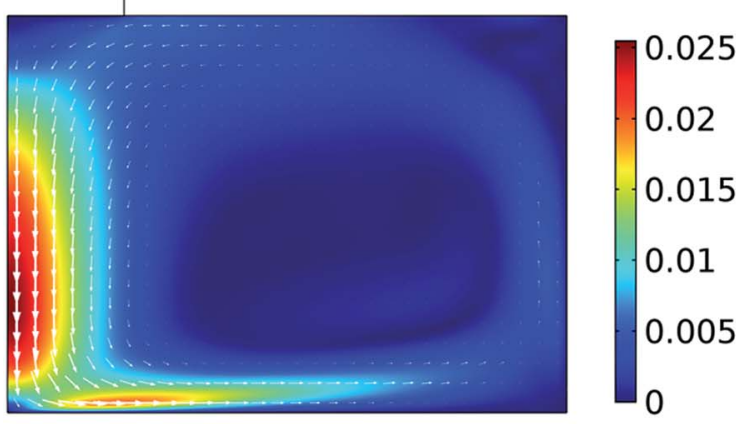

$\nabla 0$

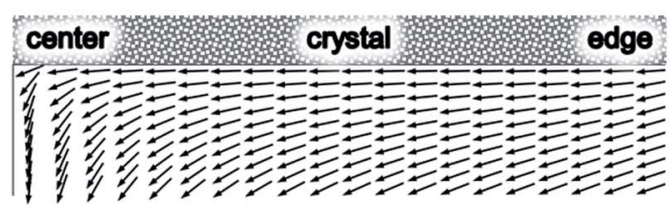

(c)
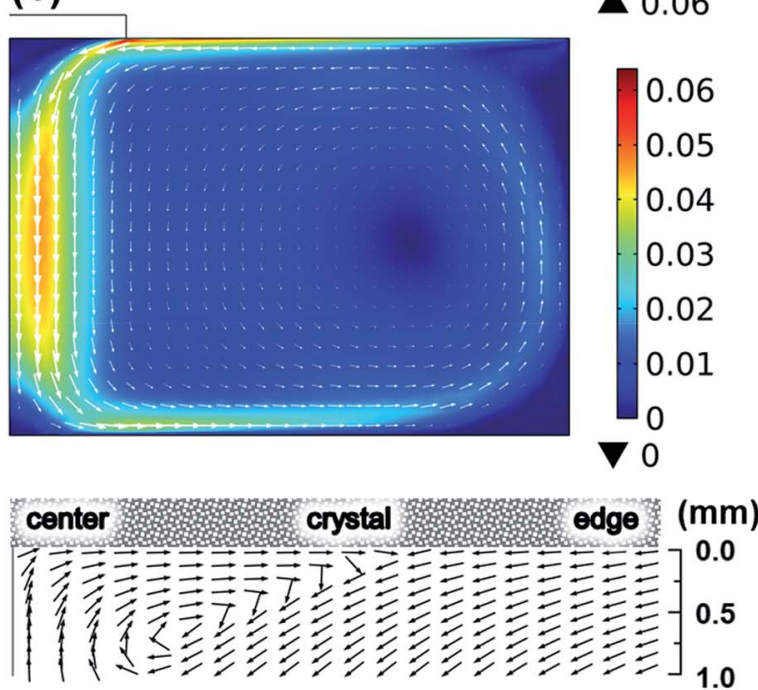

(b)

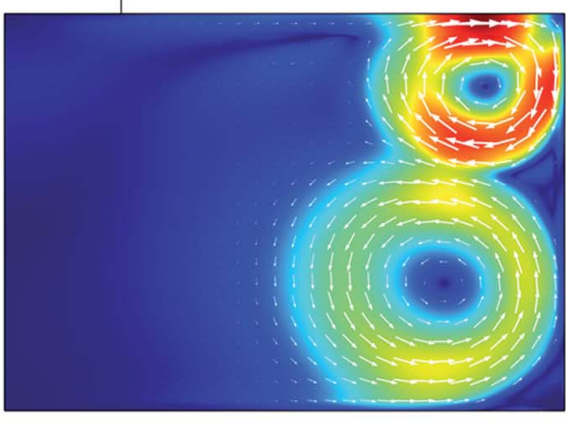

$\mathbf{\Delta} 0.202$

0.2

0.18

0.16

0.14

0.12

0.1

0.08

0.06

0.04

0.02

0

0

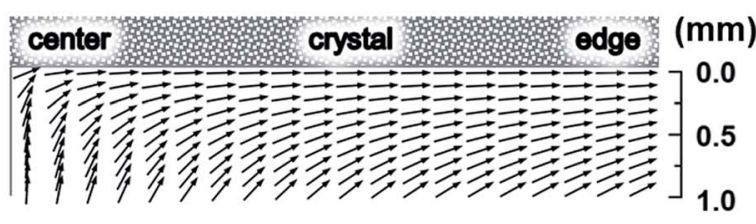

(d)

$\triangle 0.016$
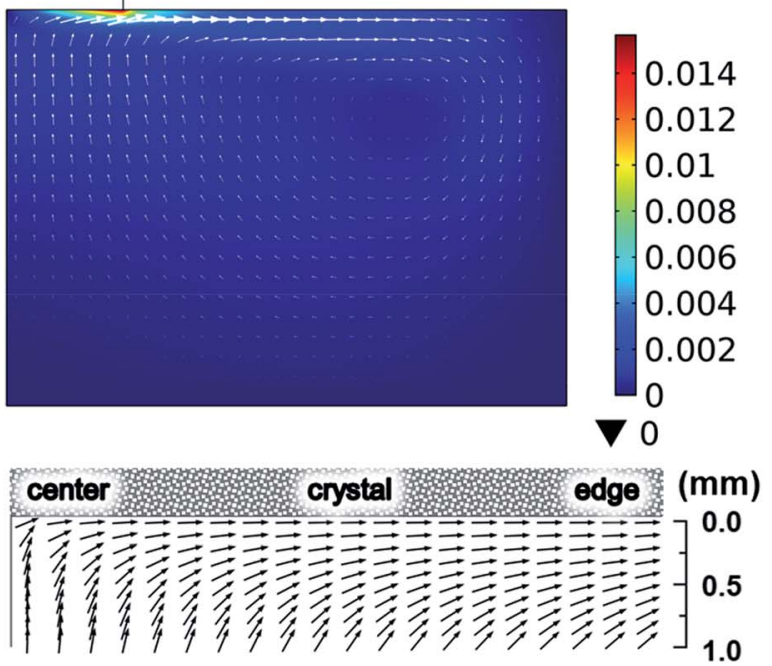

Fig. 4 Velocity fields in the entire melt and near the melt-crystal interface in simulation cases in which a single convection mechanism was considered: (a) buoyancy convection, (b) electromagnetic convection, (c) Marangoni convection, and (d) centrifugal forced convection. 
To verify the mass transport in the melt, melting experiments with $90 \% \mathrm{Si}-10 \% \mathrm{Cr}$ was performed without seed crystal at $\sim 1800{ }^{\circ} \mathrm{C}$, and the melt after cooling was evaluated with cross-sectional images using field emission scanning electron microscopy (FE-SEM). Considering the existence of $\mathrm{Cr}$ in the melt, the dynamic viscosity of the melt was adjusted to $1.0 \times$ $10^{-3}$ Pa $s$ from the relationship of the mixture viscosity, $\ln \mu_{\text {mix }}$ $=x_{\mathrm{i}} \ln \mu_{\mathrm{i}}+x_{\mathrm{j}} \ln \mu_{\mathrm{j}}$, where $5.7 \times 10^{-3} \mathrm{~Pa}$ s for $\mu_{\mathrm{Cr}}$ and $0.8 \times 10^{-3}$ Pa $\mathrm{s}$ for $\mu_{\mathrm{Si}}$.

Fig. 3 shows the simulation results obtained from the $\mathrm{Si}-\mathrm{Cr}$ melt and pure Si melt together with the experimental results of the cross-sectional FE-SEM images for the solidified melt. The bright region and dark region in the FE-SEM images were identified as Cr-rich and Si-rich regions, respectively, by energy dispersive spectroscopy (see ESI $\dagger$ ). By adding $\mathrm{Cr}$ in the Si melt, the characteristic of the fluid flow could be analyzed from the shape and size of the distinguished Cr-rich precipitates. As shown in Fig. 3c, the shape and size of the precipitates were different depending on the sampling points. While the widely spread precipitates were found in (1) and (3), the less spread precipitates were found in (2) and (4). The widely spread precipitates and the less spread precipitates were assumed to be the evidence of the rapid fluid flow and the slow fluid flow, respectively. The distribution of the precipitates correlated very well to simulation results showing the flow velocity shown in Fig. 3a. Therefore, the current simulation model used in this study was verified successfully by the melting test with $\mathrm{Si}-\mathrm{Cr}$ melt.

To determine the governing force developing fluid flow, the contribution of convection mechanisms was evaluated, as shown in Fig. 4. Compared to the fluid flow considering all convection mechanisms shown in Fig. 3, the fluid flows shown in Fig. 4 represented the individual flows originated from every convection mechanism by neglecting other convection mechanisms. Fig. 4 shows the global fluid in this study that is determined by electromagnetic convection. The fluid flow driven by electromagnetic convection indicated the maximum flow rate of $0.202 \mathrm{~m} \mathrm{~s}^{-1}$ at the melt surface near the wall of the crucible, while the maximum flow rates driven by buoyancy convection were only less than $0.025 \mathrm{~m} \mathrm{~s}^{-1}$. For the Marangoni convection and the centrifugal forced convection, the driven fluid flow reached $0.06 \mathrm{~m} \mathrm{~s}^{-1}$ and $0.016 \mathrm{~m} \mathrm{~s}^{-1}$ near the melt surface, respectively. Hence, the global fluid flow was assumed to be determined by electromagnetic convection. However, as
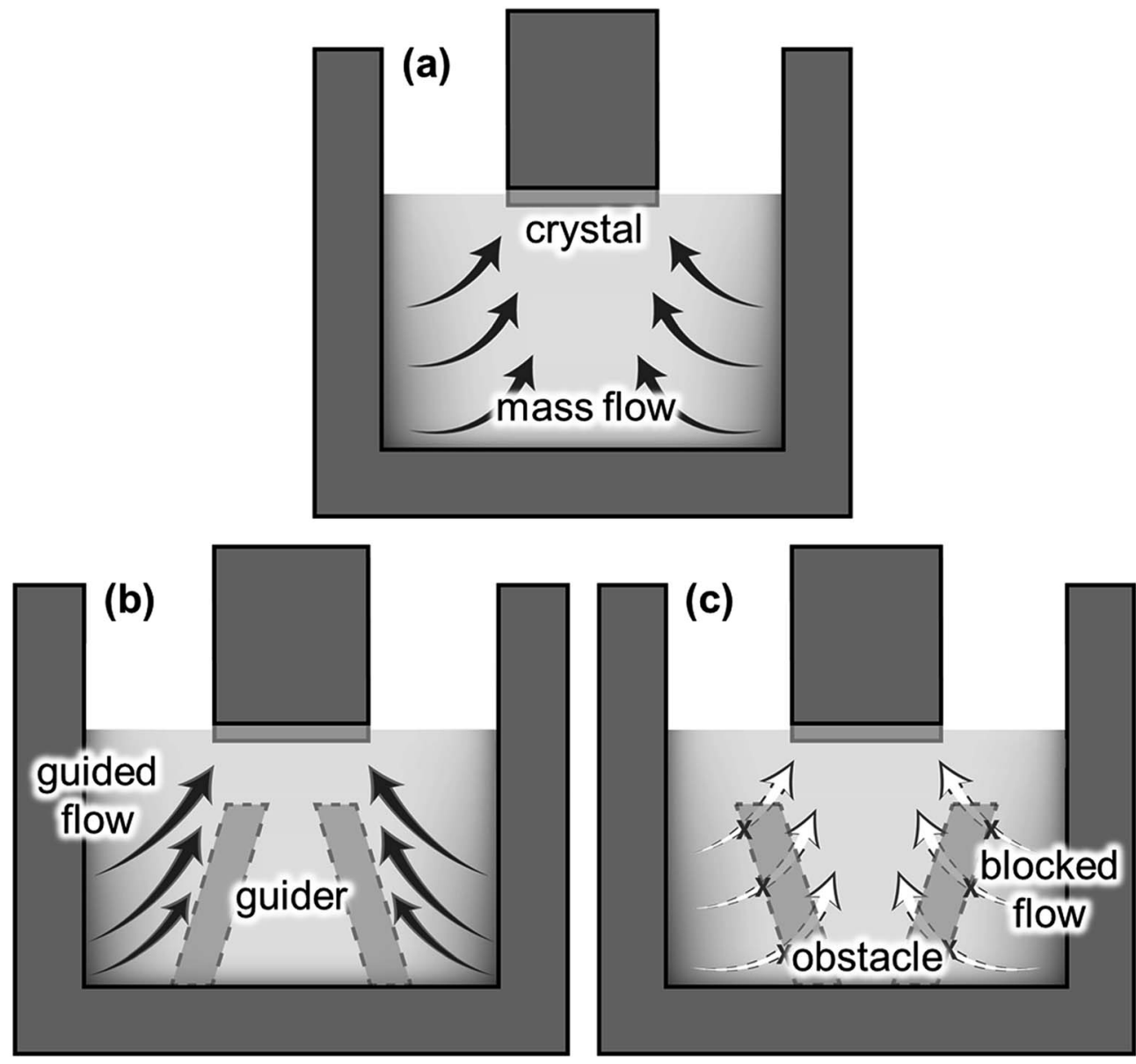

Fig. 5 (a) Carbon transportation in the conventional crucible, (b) proper implementation of a guider can guide the carbon flow toward to the seed crystal effectively, but (c) a different implement could act as an obstacle that prevents the carbon flows from distributing to the whole solution. 
reported in our previous study, ${ }^{17-20}$ the crystal growth is affected significantly by the subsurface flow near the seed crystal. This suggests that the Marangoni convection and centrifugal forced convection had also contributed to the crystal growth together with electromagnetic convection. The normalized velocity field shown in Fig. 4 shows that the fluid flow beneath the crystal is determined by the interaction driven by electromagnetic convection, Marangoni convection, and centrifugal forced convection.

\section{Mass transport control by flow modifier}

In the previous section, we confirmed that the global fluid flow was governed by the electromagnetic convection that formed strong convection near the crucible wall. Hence, the dissolved C near the crucible wall could be transported to the SiC crystal, as illustrated in Fig. 5a. The structural modification of the reservoir affects the fluid flow significantly, as represented by the "immersion guide", ${ }^{14}$ to promote vertical fluidic flow to enhance the growth rate of SiC in the TSSG method. In this study, the fluid flow near the crystal is along the reverse radial direction from the crucible wall to the crystal. Hence, the installation of the FM on the bottom of the crucible is an easy method to control the fluid flow of TSSG, as represented in Fig. $5 \mathrm{~b}$ and c. If the FM serves as a fluid guide to enhance the mass transport of $\mathrm{C}$, the dissolved $\mathrm{C}$ is consumed more efficiently to grow $\mathrm{SiC}$ on the crystal. Alternatively, the FM serves as an obstacle to block the mass transport of $\mathrm{C}$ to the crystal. In this section, the effect of FM installation is investigated with the verified simulation model.

Fig. 6 shows the fluid velocity field, temperature distribution, and carbon concentration distribution in the melt at $\sim 1800{ }^{\circ} \mathrm{C}$ with the melt surface temperature in the cases shown in Fig. 5. In the fluid velocity fields evaluated in the three cases, two primary vortexes appeared near the crucible wall. In all cases, the upper vortexes were always stronger than the lower vortexes, thus suggesting that the upper vortex primarily take responsibility to the mass transport of the melt in the TSSG method. In the case of no-FM, the upper vortex was smaller than the lower vortex, and the two vortexes were connected to each other. The coupling of two vortexes can enhance the uniformity of the temperature and C concentration. In the case of FM-upward, however, two vortexes were separated, such that the dissolved carbon was confined at the corner crucible, so the heat and mass transfer via the lower vortex was minimized. In the case of FM-downward, the upper vortex was large, and two vortexes were connected as in the case of no-FM. Furthermore, the fluid flow was guided along the FM slope and toward the crystal. Therefore, the heat and mass were transported well to the crystal in the following order: FM-downward > no-FM > FMupward. With the model, FM was confirmed to be effective in enhancing the mass transport of $\mathrm{C}$ to the crystal, especially in the case of FM-downward.

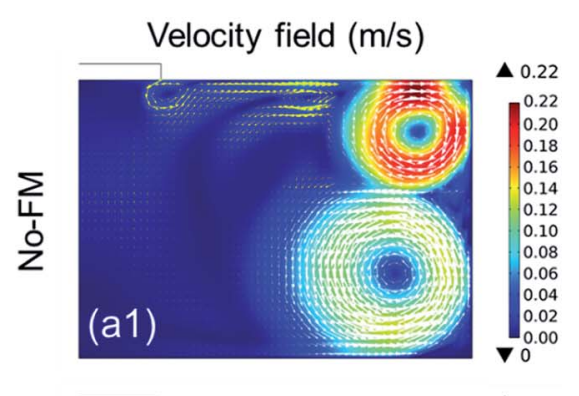

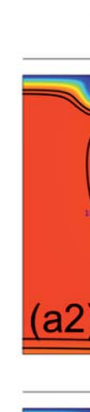

Temperature $\left({ }^{\circ} \mathrm{C}\right)$
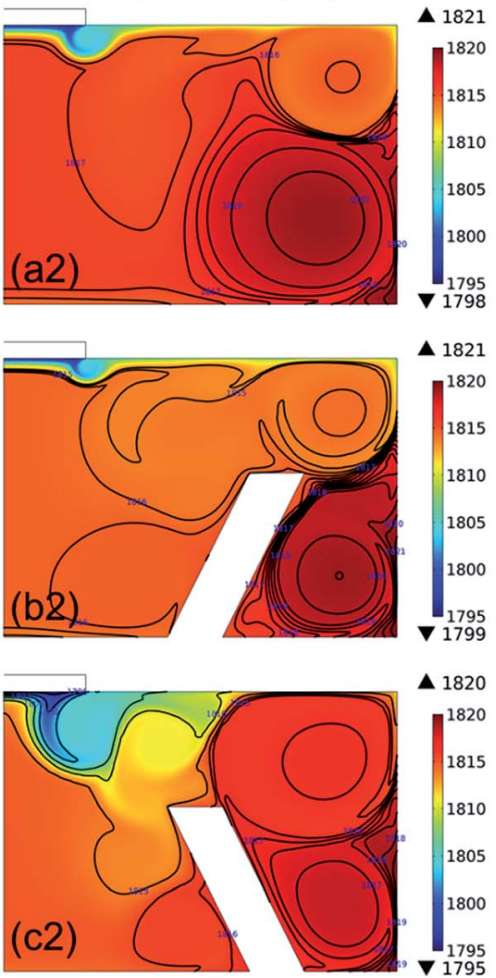

Carbon concentration $\left(\mathrm{mol} / \mathrm{m}^{3}\right)$

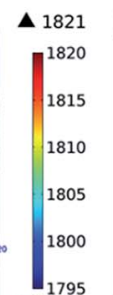

(1)
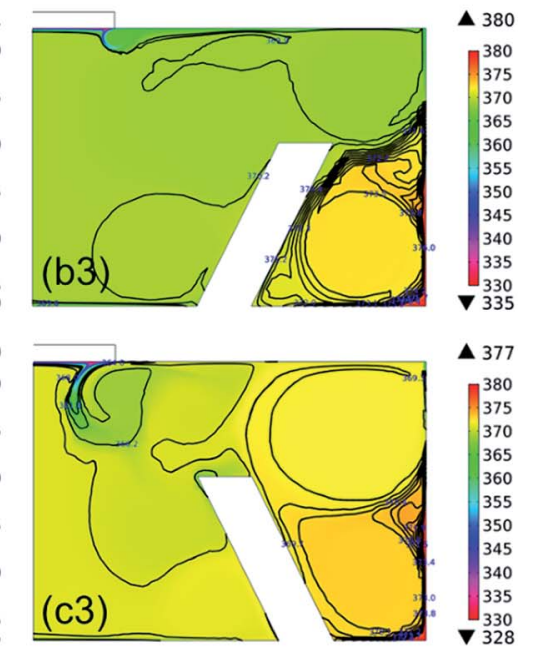

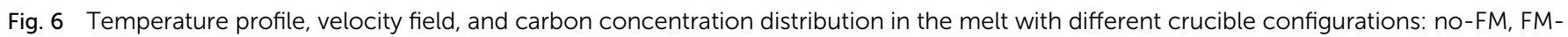
upward, and FM-downward. 
Compared to previous studies ${ }^{\mathbf{1 3 , 1 4}}$ on the flow modification, the fluid flow controlled by FM in this study represents remarkable differences. Mercier et al. proposed several crucible configurations, including a "podium crucible" minimizing the effect of electromagnetic convection and Marangoni convection to the melt flow near the growth front. ${ }^{13}$ Kusunoki et al. reported the homogeneity of the fluid flow under the seed crystal improved by placing a ring inside the melt, so-called an "immersion guide (IG)". ${ }^{14}$ With IG, the upper region of the melt had a relatively higher temperature, $\mathrm{C}$ solubility, and $\mathrm{C}$ concentration compared to the lower region so that the crystal growth rate increased. In contrary, the "flow modifier (FM)" attached to the bottom of the crucible in this study, acted as a flow guide forming two flow vortexes connected each other which directed the mass transport of $\mathrm{C}$ from the crucible wall to the seed crystal. Since FM enables the efficient use of $\mathrm{C}$ under uniform temperature gradient by forming the flow guide, the application of FM is advantageous in the long term crystal growth which requires the homogeneous process less dependent on growing time. The application of FM to long-term growth will be studied in our next work.
The crystal growth of SiC by modifying mass transport using the FM was investigated experimentally between $\sim 1700$ and $\sim 1900{ }^{\circ} \mathrm{C}$. Fig. 7a, b, and c represent the change in growth rate as a function of the melt surface temperature for the cases of noFM, FM-upward, and FM-downward, respectively. From the Raman spectroscopy shown in Fig. 7d, the grown layers in all conditions were identified as pure $4 \mathrm{H}$-SiC because the Raman peaks demonstrated the characteristic peaks of $\mathrm{SiC}$ at $\sim 776$, $\sim 796$, and $\sim 964 \mathrm{~cm}^{-1}$, respectively. The growth rates increased with temperature. It is noteworthy that the growth rate indicated the highest values for FM-downward at all temperatures and the lowest values for FM-upward, as expected in the simulations. Compared to no-FM, FM-downward increased the growth rate as much as $78.46 \%, 44.08 \%$, and $18.67 \%$ at $1700{ }^{\circ} \mathrm{C}$, $1800{ }^{\circ} \mathrm{C}$, and $1900{ }^{\circ} \mathrm{C}$, respectively, which proved that the proper FM configuration could improve the growth rate in TSSG, especially at low temperatures.

To estimate the growth rate with the simulation model, the $\mathrm{C}$ supersaturation and $\mathrm{C}$ flux to the crystal were considered. The $\mathrm{C}$ supersaturation is defined as $S=\left(c-C_{0}\right) / C_{0}$. Because the growth rate is directly correlated with the $\mathrm{C}$ supersaturation near the crystal,
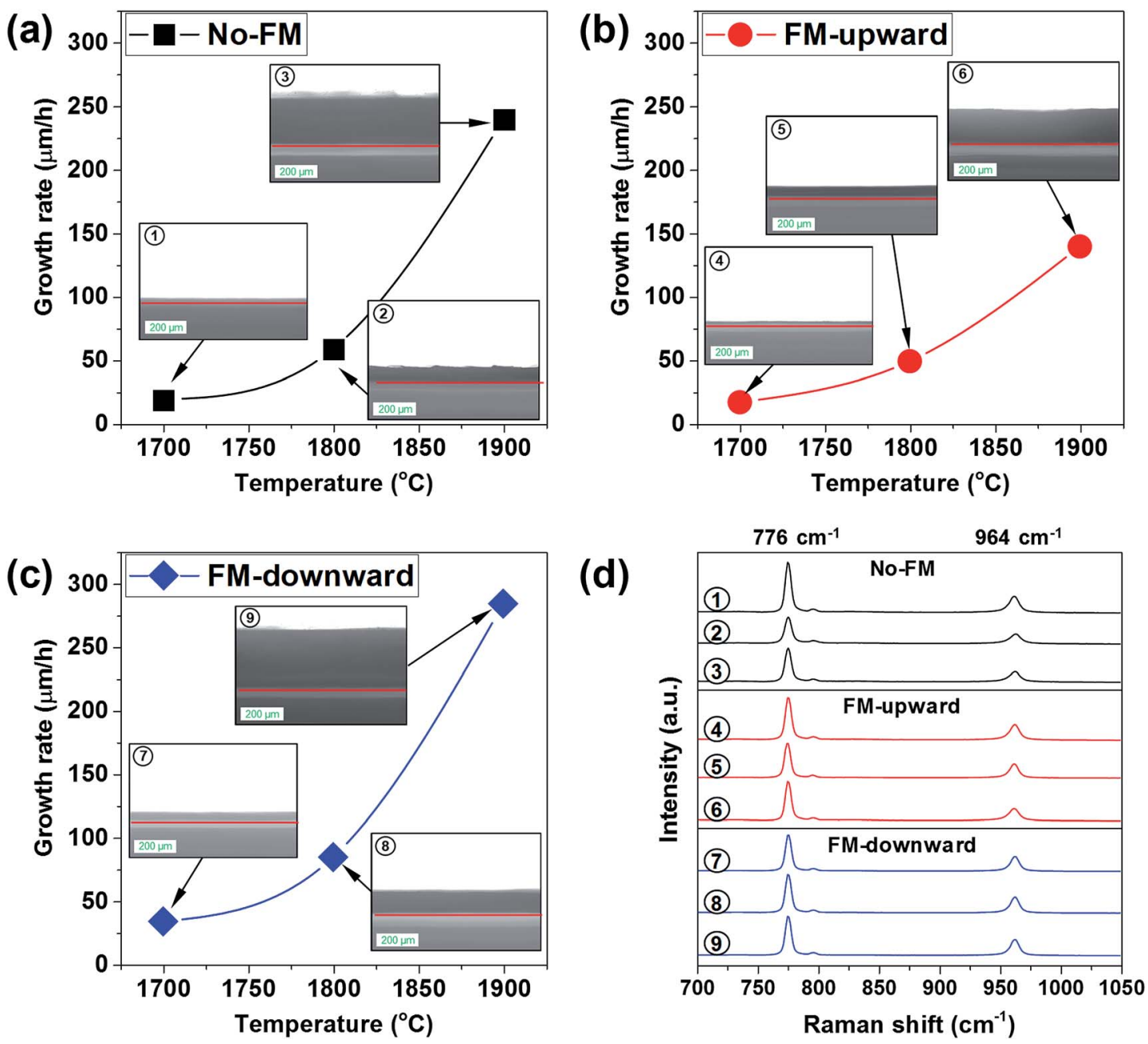

Fig. 7 (a-c) Cross-sectional optical images and growth rates of grown crystals at different FM configurations and temperatures, and (d) the micro-Raman spectra of grown crystals. 
the calculated C supersaturation along the distance from the crystal/ melt interface was plotted for the cases of no-FM, FM-upward, and FM-downward at $\sim 1800{ }^{\circ} \mathrm{C}$ in Fig. 8a. The $\mathrm{C}$ supersaturation indicated maximum values at $\sim 0.2 \mathrm{~mm}$ from the crystal, thus suggesting a mass transport model of accumulation and the consumption of C. As shown in Fig. 8a, the melt with supersaturated $\mathrm{C}$ near the crystal can be divided to two regions. In the $\mathrm{C}$ accumulation region, the $\mathrm{C}$ supersaturation increases as $\mathrm{C}$ approaches the crystal by mass transport. In the $\mathrm{C}$ consumption region, the $\mathrm{C}$ supersaturation decreases as $\mathrm{C}$ approaches the crystal because $\mathrm{C}$ consumption contributes to the crystal growth. The $\mathrm{C}$ accumulation region and $\mathrm{C}$ consumption region are divided at the depth with the maximum $\mathrm{C}$ concentration, as shown in Fig. 8a. With this model, the $\mathrm{C}$ contributing to crystal growth is assumed to be limited to the supersaturated $\mathrm{C}$ in the $\mathrm{C}$ consumption region.

The $\mathrm{C}$ diffusive flux to the seed crystal $J_{\mathrm{c}}$ is derived from Fick's law:

$$
J_{\mathrm{c}}=-D \frac{\delta c}{\delta x}=-D \frac{\delta(S+1) C_{0}(T)}{\delta x} .
$$

If the temperature gradient in the consumption region is negligible, the diffusion coefficient $D$ and solubility $C_{0}(T)$ are constant. Subsequently, the C diffusive flux to the crystal is proportional to the $\mathrm{C}$ supersaturation gradient $(S+1) / \delta x$, where $x$ is the distance from the growth front. As shown in Fig. 8a, the C supersaturation is the highest for FM-downward, while the $\mathrm{C}$ supersaturations for no-FM and FM-upward are in the same order. Therefore, the $\mathrm{C}$ diffusive flux for of FM-downward is the highest; therefore, the growth rate in that case is estimated to be the highest among the suggested FM configurations.

However, an abrupt temperature gradient occurred near the crystal owing to the heat transfer to the shaft and melt; ${ }^{20}$ therefore, the carbon solubility $C_{0}(T)$ is not constant. Furthermore, the convective $\mathrm{C}$ flux at the consumption region cannot to be ignored. Hence, to better derive the growth rate, the total $\mathrm{C}$ flux normal to the crystal including the diffusive flux and convective flux with temperature gradient near the crystal was calculated numerically, as shown in Fig. 8b. The total C flux normal to the crystal was proportional to the growth temperature, and the highest C flux was obtained for FM-downward, followed by no-FM and FM-upward.

Because the surface reaction in the crystal growth of SiC occurred at high temperatures, the kinetics in the surface reaction at the crystal can be ignored to determine the growth
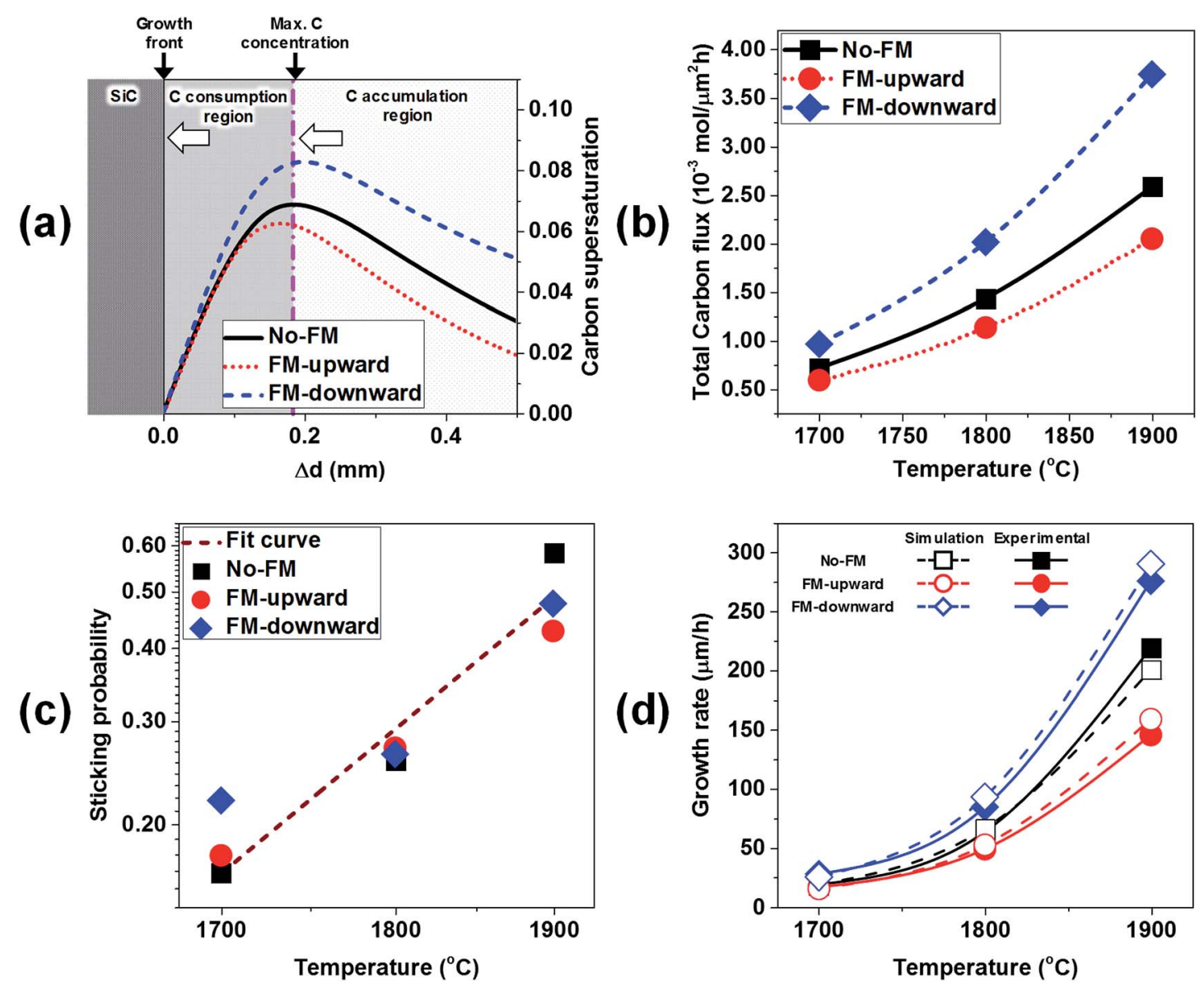

Fig. 8 (a) $\mathrm{C}$ supersaturation distribution in the melt versus distance from the melt/crystal interface at $1800^{\circ} \mathrm{C}$ and different $\mathrm{FM}$ configurations, (b) total $\mathrm{C}$ flux approaching the crystal surface at different temperatures and FM configurations, (c) fitted sticking probability of $\mathrm{C}$ atoms on growth front, and (d) simulated and experimental growth rate at $1700{ }^{\circ} \mathrm{C}, 1800^{\circ} \mathrm{C}$, and $1900{ }^{\circ} \mathrm{C}$. 
rate. ${ }^{16}$ Hence, by neglecting the kinetics limitation in the growth reaction, the growth rate of $\mathrm{SiC}$ crystal is derived as

$$
G=\frac{M_{\mathrm{Si}}}{\rho \pi r^{2}} \iint_{S} F \mathrm{~d} S,
$$

where $F(r)$ is the total $\mathrm{C}$ flux normal to the seed crystal $S$. However, the growth rates derived from eqn (12) were remarkably higher than the experimental values shown in Fig. 7a-c. To match the simulated growth rates to the experimental values, the effective $\mathrm{C}$ atoms contributing to the crystal growth is considered by adopting the adsorption rate. The adsorption rate $R_{\text {ads }}$ of C on the crystal is derived by the following equation:

$$
R_{\mathrm{ads}}=P_{\mathrm{s}} F,
$$

where $P_{\mathrm{s}}$ is the sticking probability of $\mathrm{C}$ atoms on the growth front. The sticking probability $P_{\mathrm{s}}$ follows the Arrhenius equation:

$$
P_{\mathrm{s}}=A \mathrm{e}^{\frac{-E_{\mathrm{a}}}{R T}},
$$

where $A$ is a constant, $E_{\mathrm{a}}$ is the adsorption energy, and $R$ is the ideal gas constant of $8.314 \mathrm{~J} \mathrm{~mol}^{-1} \mathrm{~K}^{-1}$. From the simulated $\mathrm{C}$ flux and the experimental growth rate, the sticking probability was obtained, as shown in Fig. 8c. From the sticking probability, the adsorption energy $E_{\mathrm{a}}$ was derived to be $194 \mathrm{~kJ} \mathrm{~mol}^{-1}$, which was similar to those in previous studies. ${ }^{16,21}$ Considering the adsorption rate, the growth rate can be obtained with the following equation:

$$
\begin{aligned}
G & =\frac{M_{\mathrm{Si}}}{\rho \pi r^{2}} \iint_{S} R_{\mathrm{ads}} \mathrm{d} S \\
& =\frac{M_{\mathrm{Si}}}{\rho \pi r^{2}} \times\left(2.2 \times 10^{4}\right) \times \exp \left(\frac{-23.33 \times 10^{3}}{T}\right) \iint_{S} F \mathrm{~d} S .
\end{aligned}
$$

Fig. 8d shows the experimental growth rates together with the simulated values obtained with eqn (15) for the cases of noFM, FM-upward, and FM-downward. Regardless of whether the FM existed, the simulation results indicated good agreement with the experimental results.

From this study, we discovered that the FM affected the growth rate of SiC using TSSG significantly and that the configuration of FM-downward was the best to enhance the growth rate at the given temperature and reactor design.

\section{Conclusion}

In this study, we discovered that the electromagnetic convection governed the global melt flow in TSSG of SiC. The other mechanisms, including Marangoni convection, buoyancy convection, and centrifugal forced convection were found to considerably influent on the fluid flow near the crystal. The modification of fluid flow by setting the FM was proved to be very strong to determine the growth rate of SiC. Especially, the FM-downward was found to be an efficient method to get high growth rate by the efficient use of the dissolved $\mathrm{C}$ in the melt at the given temperature and reactor design.

\section{Author contributions}

The manuscript was written through the contributions of all authors. All authors have given approval to the final version of the manuscript.

\section{Conflicts of interest}

The authors declare no competing financial interest.

\section{Acknowledgements}

This study was financially supported by the Strategic Core Materials Technology Development program (No. 10062272) funded by the Ministry of Trade, Industry, and Energy (MOTIE), Korea. The authors thank Mr Dae-Seop Byeon (KICET) for his help and fruitful discussion.

\section{References}

1 Y. M. Tairov and V. F. Tsvetkov, Investigation of Growth Processes of Ingots of Silicon Carbide Single Crystals, J. Cryst. Growth, 1978, 43(2), 209-212.

2 A. R. Powell, J. J. Sumakeris, Y. Khlebnikov, M. J. Paisley, R. T. Leonard, E. Deyneka, S. Gangwal, J. Ambati, V. Tsevtkov, J. Seaman, et al., Bulk Growth of Large Area SiC Crystals, Mater. Sci. Forum, 2016, 858, 5-10.

3 D. H. Hofmann and M. H. Müller, Prospects of the Use of Liquid Phase Techniques for the Growth of Bulk Silicon Carbide Crystals, Mater. Sci. Eng., B, 1999, 61-62, 29-39.

4 K. Kamei, K. Kusunoki, N. Yashiro, N. Okada, K. Moriguchi, H. Daikoku, M. Kado, H. Suzuki, H. Sakamoto and T. Bessho, Crystallinity Evaluation of $4 \mathrm{H}-\mathrm{SiC}$ Single Crystal Grown by Solution Growth Technique Using Si-Ti-C Solution, Mater. Sci. Forum, 2012, 717-720, 45-48.

5 K. Kusunoki, K. Kamei, Y. Ueda, S. Naga, Y. Ito, M. Hasebe, T. Ujihara and K. Nakajima, Crystalline Quality Evaluation of $6 \mathrm{H}$-SiC Bulk Crystals Grown from Si-Ti-C Ternary Solution, Mater. Sci. Forum, 2005, 483-485, 13-16.

6 S. Harada, Y. Yamamoto, K. Seki, A. Horio, M. Tagawa and T. Ujihara, Different Behavior of Threading Edge Dislocation Conversion during the Solution Growth of $4 \mathrm{H}-$ SiC Depending on the Burgers Vector, Acta Mater., 2014, 81, 284-290.

7 S. Harada, Y. Yamamoto, K. Seki, A. Horio, T. Mitsuhashi, M. Tagawa and T. Ujihara, Evolution of Threading Screw Dislocation Conversion during Solution Growth of $4 \mathrm{H}-\mathrm{SiC}$, APL Mater., 2013, 1(2), 022109.

8 S. Xiao, S. Harada, K. Murayama, M. Tagawa and T. Ujihara, Conversion Behavior of Threading Screw Dislocations on $\mathrm{C}$ Face with Different Surface Morphology During 4H-SiC Solution Growth, Cryst. Growth Des., 2016, 16(11), 64366439.

9 J. E. Lee, B. G. Kim, J.-Y. Yoon, M.-T. Ha, M.-H. Lee, Y. Kim, W.-S. Seo, H.-J. Choi, W.-J. Lee and S.-M. Jeong, The Role of an $\mathrm{SiC}$ Interlayer at a Graphite-Silicon Liquid Interface in the 
Solution Growth of SiC Crystals, Ceram. Int., 2016, 42(10), 11611-11618.

10 T. Yamamoto, N. Adkar, Y. Okano, T. Ujihara and S. Dost, Numerical Investigation of the Transport Phenomena Occurring in the Growth of SiC by the Induction Heating TSSG Method, J. Cryst. Growth, 2017, 474, 50-54.

11 T. Yamamoto, Y. Okano, T. Ujihara and S. Dost, Global Simulation of the Induction Heating TSSG Process of SiC for the Effects of Marangoni Convection, Free Surface Deformation and Seed Rotation, J. Cryst. Growth, 2017, 470, 75-88.

12 F. Mercier and S. Nishizawa, Solution Growth of SiC from Silicon Melts: Influence of the Alternative Magnetic Field on Fluid Dynamics, J. Cryst. Growth, 2011, 318(1), 385-388.

13 F. Mercier, J.-M. Dedulle, D. Chaussende and M. Pons, Coupled Heat Transfer and Fluid Dynamics Modeling of High-Temperature SiC Solution Growth, J. Cryst. Growth, 2010, 312(2), 155-163.

14 K. Kusunoki, N. Okada, K. Kamei, K. Moriguchi, H. Daikoku, M. Kado, H. Sakamoto, T. Bessho and T. Ujihara, Top-Seeded Solution Growth of Three-Inch-Diameter 4H-SiC Using Convection Control Technique, J. Cryst. Growth, 2014, 395, 68-73.

15 F. Durand and J. C. Duby, Carbon Solubility in Solid and Liquid Silicon-A Review with Reference to Eutectic Equilibrium, J. Phase Equilib., 1999, 20(1), 61-63.
16 J. Lefebure, J.-M. Dedulle, T. Ouisse and D. Chaussende, Modeling of the Growth Rate during Top Seeded Solution Growth of SiC Using Pure Silicon as a Solvent, Cryst. Growth Des., 2012, 12(2), 909-913.

17 K. Ariyawong, Y. J. Shin, J.-M. Dedulle and D. Chaussende, Analysis of Macrostep Formation during Top Seeded Solution Growth of 4H-SiC, Cryst. Growth Des., 2016, 16(6), 3231-3236.

18 H. Daikoku, M. Kado, A. Seki, K. Sato, T. Bessho, K. Kusunoki, H. Kaidou, Y. Kishida, K. Moriguchi and K. Kamei, Solution Growth on Concave Surface of $4 \mathrm{H}-\mathrm{SiC}$ Crystal, Cryst. Growth Des., 2016, 16(3), 1256-1260.

19 C. Zhu, S. Harada, K. Seki, H. Zhang, H. Niinomi, M. Tagawa and T. Ujihara, Influence of Solution Flow on Step Bunching in Solution Growth of SiC Crystals, Cryst. Growth Des., 2013, 13(8), 3691-3696.

20 M.-T. Ha, Y.-J. Shin, M.-H. Lee, C.-J. Kim and S.-M. Jeong, Effects of the Temperature Gradient Near the Crystal-Melt Interface in Top Seeded Solution Growth of SiC Crystal, Phys. Status Solidi A, 2018, 215(20), 1701017.

21 M. Nasir Khan, S. Nishizawa, W. Bahng and K. Arai, LiquidPhase Epitaxy on 6H-SiC Acheson Seed Crystals in Closed Vessel, J. Cryst. Growth, 2000, 220(1-2), 75-81. 